\title{
Optimization of system's parameters for wavelength conversion of E-band signals
}

\author{
Yazan Alkhlefat, Sevia Mahdaliza Idrus, Farabi M. Iqbal \\ Department of Electrical Engineering, Universiti Teknologi Malaysia, Johor Bahru, Malaysia
}

\begin{abstract}
Article Info
Article history:

Received Jun 21, 2021

Revised Jul 5, 2021

Accepted Aug 4, 2021

Keywords:

$5 \mathrm{G}$

Millimeter waves

OFDM-SPM

Semiconductor optical amplifier

Wavelength conversion

ABSTRACT

Current and future wireless communication systems are designed to achieve the user's demands such as high data rate and high speed with low latency and simultaneously to save bandwidth and spectrum. In 5G and $6 \mathrm{G}$ networks, a high speed of transmitting and switching is required for internet of things (IOT) applications with higher capacity. To achieve these requirements a semiconductor optical amplifier (SOA) is considered as a wavelength converter to transmit a signal with an orthogonal frequency division multiplexing with subcarrier power modulation (OFDM-SPM). It exploits the subcarrier's power in conventional OFDM block in order to send additional bits beside the normally transmitted bits. In this paper, we optimized the SOA's parameters to have efficient wavelength conversion process. These parameters are included the injection current (IC) of SOA, power of pump and probe signals. A 7 Gbps OFDM-SPM signal with a millimeter waves (MMW) carrier of $80 \mathrm{GHz}$ is considered for signal switching. The simulation results investigated and analyzed the performance of the designed system in terms of error vector magnitude (EVM), bit error rate (BER) and optical signal-to-noise ratio (OSNR). The optimum value of IC is $0.6 \mathrm{~A}$ while probe power is 9.45 and $8.9 \mathrm{dBm}$ for pump power. The simulation is executed by virtual photonic integrated (VPI) software.
\end{abstract}

This is an open access article under the $\underline{C C B Y-S A}$ license.

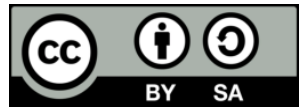

\section{Corresponding Author:}

Sevia Mahdaliza Idrus

Department of Electrical Engineering, Universiti Teknologi Malaysia

81310, Johor Bahru, Johor, Malaysia

Email: sevia@utm.my

\section{INTRODUCTION}

The huge demands on high capacity and bandwidth (BW) interactive applications such as virtual reality (VR) and autonomous vehicles are considered as the main motivators to find the next generations of wireless technologies. The standardization of the current fifth-generation $(5 \mathrm{G})$ is driven by the enhancement of millimeter-wave (MMW) technology [1]. Additionally, in 5G mobile networks, the integration of advanced technologies in access techniques, data modulation, frequency bands, and antenna designs helps to achieve the user's demands [2], [3] However, MMW signals which provide higher bandwidth and data rate (Gbps) suffer from power drop due to the attenuation in a high-frequency band which leads to difficulties of new mobile technology connectivity. This issue can be resolved by integration of photonics and MMW using the concept of millimeter-wave-over-fiber (MMWoF) [4]-[6] as shown in Figure 1 [7]. Moreover, the features of photonic components and devices can be exploited in order to establish different types of electronic systems Photonic devices are playing a key role in switching and routing because it provides main requirements of all wavelength converters (WCs) and optical switches such as low latency, high bandwidth, flexibility, and transparency [7]. One type of wavelength converters can be used based on a semiconductor 
optical amplifier (SOA) by exploiting its nonlinear effects; self-phase modulation (SPM), cross gain modulation (XGM), and four-wave mixing (FWM) [7].

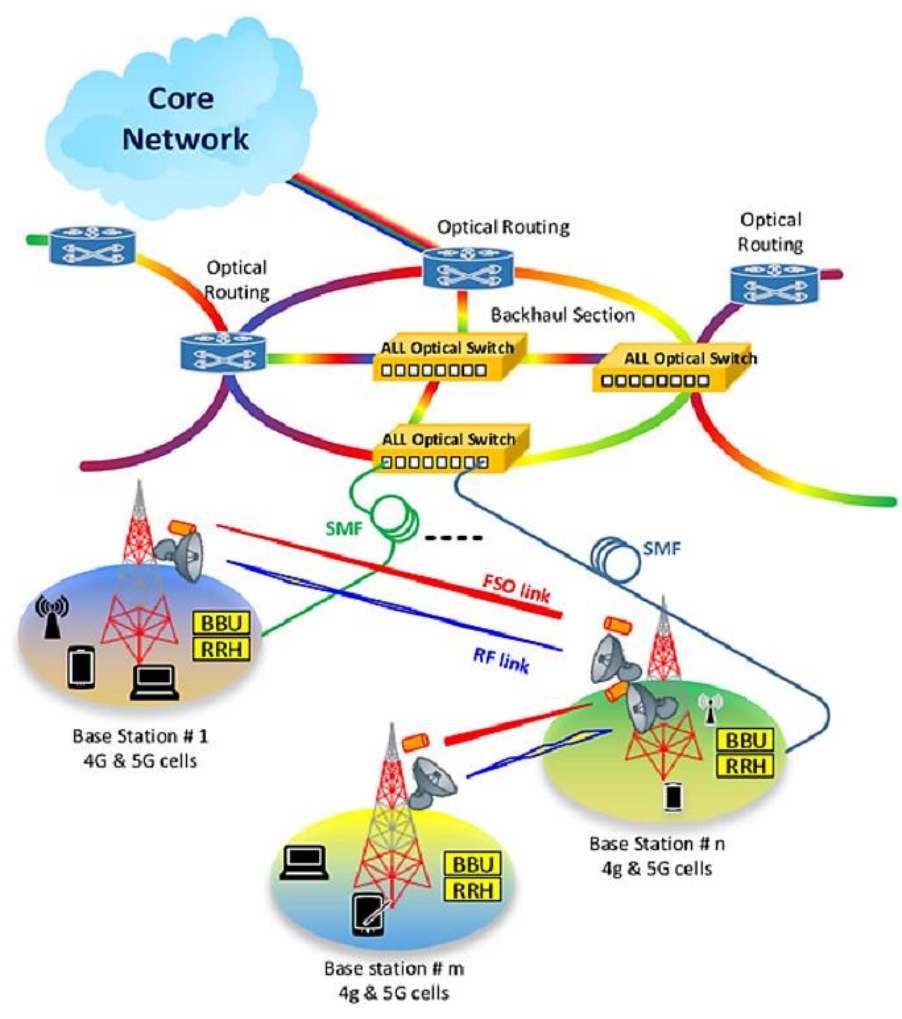

Figure 1. Architecture photonic switching with millimeter-wave over fiber: radio frequency (RF), free-space optical (FSO), remote radio head (RRH), baseband unit (BBU), and single-mode fiber (SMF) [7]

Moreover, the 5G wireless networks have been designed and studied to achieve the demand for higher bandwidth and data rates, so it should find a suitable modulation format that can be used to save bandwidth in current-generation $5 \mathrm{G}$ and next-generation $6 \mathrm{G}$ and beyond. In previous generations like 4G, orthogonal frequency division multiplexing (OFDM) has been used to achieve higher data rates [8]. On the other hand, it is suffering from a high peak-to-average power ratio (PAPR), so the authors in [9] proposed a new method including modified tone reservation to mitigate PAPR value with low complexity of implementation compared with classical tone reservation (TR) method. Moreover, Abid et al. [10] proposed another technique to reduce PAPR which uses discrete cosine transform (DCT), low-density parity-check code (LDPC), and $\mu$-law companding. Additionally, linear amplifiers are used in orthogonal frequency division multiplexing (OFDM) to ensure that the PAPR is accommodated and no distortions are resulted in the output signals [11]. Hajar et al. [12] proposed a new modulation scheme which is called orthogonal frequency division multiplexing with subcarrier power modulation (OFDM-SPM). It exploits the subcarrier's power in conventional OFDM block in order to send additional bits beside the normally transmitted bits.

Due to the aforementioned advantages of switching and wavelength conversion using SOA, the authors paid more interest in this regard. Saadaoui et al. [13] implemented a photonic system based on MMW switching experimentally for quadrature phase shift keying (QPSK) signal with carrier frequency $30 \mathrm{GHz}$ to carry 3Gbaud data rate. While in [14] demonstrated experimentally a system with a 16-QAM signal to transmit $200 \mathrm{Mbps}$ at the carrier frequency of $20 \mathrm{GHz}$. In addition, Li et al. [15] studied the system of an ultra-high-speed transmission based on all-optical wavelength conversion to transmit the bit rate of 227 Gbps with a modulation format of 128-QAM using SOA. Alkhlefat et al. [16] compared between universal filtered multi carrier (UFMC) and filter bank multi-carrier (FBMC) in the wavelength conversion using SOA and proposed the recommended parameters which provide an efficient conversion. In [17], a wavelength conversion of QPSK signal with $9 \mathrm{Gbps}$ data rate and $30 \mathrm{GHz}$ carrier frequency has been investigated using OptiSystem software. The results show that the spacing between pump and probe wavelength is equal to 1.6 nm. Lee and Song [18] implemented experimentally an radio-over-fiber (RoF) downlink by using the up- 
conversion technique in SOA for a cross-polarization modulation (XPolM) in order to transmit 16-QAM OFDM signal with $10 \mathrm{Gbps}$ and $60 \mathrm{GHz}$ carrier frequency. In [19], MMW signal 16-QAM OFDM with $30 \mathrm{GHz}$ carrier frequency and $10 \mathrm{Gbps}$ data rate was implemented using simulation via VPI software and then the performance was analyzed.

In this paper, a wavelength conversion system and MMW switching using SOA for a signal of OFDM-SPM, which is well-appropriate for $5 \mathrm{G}$ and $6 \mathrm{G}$ communication networks is proposed. Firstly, a simulation model has been established in the software of VPI transmission Maker to convert the OFDM-SPM signal from one wavelength to another one and to optimize the SOA's parameters to obtain the high system performance in terms of optical signal-to-noise ratio (OSNR), error vector magnitude (EVM), and bit error rate $(\mathrm{BER})$

The remaining sections of the paper are ordered: in section 2, we discuss and describe the proposed modulation format OFDM-SPM and how it works. Moreover, section 2 shows how the signal is traveling through SOA. Sections 3 and 4 display the simulation model and the simulation results, respectively. Section 5 provides the conclusion of the results.

\section{RESEARCH METHOD}

In this section, we describe the system model of OFDM-SPM which is considered a suitable modulation technique for next generation networks in terms of transmitter and receiver. Then we describe the theoretical model for SOA and the travelling wave through it.

\subsection{OFDM-SPM}

In recent researches, there are additional modulation techniques that are exploiting the third dimension together with an existing 2-D signal plan in order to send additional information. The third dimension can be used based on the application, its capabilities, and requirements. Examples of these new emerging modulation techniques are OFDM-SPM, OFDM-index modulation (OFDM-IM), spatial modulation orthogonal frequency division multiplexing (SM-OFDM), OFDM with pulse superposition modulation (OFDM-PSM), and OFDM with subcarrier number modulation (OFDM-SNM) [20].

In this work we will use a modulation scheme that is suitable to be used in next-generation networks; it is called OFDM-SPM. We will use it in MMW switching and wavelength conversion using SOA and optimize the SOA's parameters to obtain higher system performance. In OFDM-SPM, the third dimension has been added in order to carry more data. This dimension is defined as the power of OFDM subcarriers and it can be used to send additional data bits while using OFDM subcarriers for transmitting modulated data symbols. Hajar et al. [12] show that the proposed OFDM-SPM provides better performance and high spectral gain than classical OFDM with binary phase-shift keying (BPSK). This is due to half number of subcarriers required in OFDM-SPM comparing with conventional OFDM with BPSK to transmit a bit sequence. Consequently, the OFDM-SPM improving the system as it doubles the spectral efficiency and throughput for high OSNR values. In addition, the transmission power in OFDM-SPM is reduced to half with a saving of spectral gain.

\subsubsection{Transmitter part}

OFDM-SPM utilizes the subcarrier's power in the symbol of classical OFDM, in order to send two or more bits per subcarrier. Figure 2(a) displays the transmitter of OFDM-SPM while Figure 2(b) displays the receiver part. In Figure 2(a), incoming bits $(2 n)$ are divided into two sets of $n$ bits, which represent the subcarrier's number in the classical OFDM block utilized to convey data. The function of the first $n$ bit is determining the short circuit (SC) power, where $i^{\text {th }}$ bit determines the $i^{\text {th }}$ SC power level which is used to carry data. A 1 and 0 are used to set the SC power to high and low respectively. The second $n$ bits are indicating to the bits which need modulation using normal BPSK modulation format. Then the symbols of BPSK are allocated to their corresponding subcarriers [12].

\subsubsection{Receiver part}

As can be seen in Figure 2(b), the most important advantage of the OFDM-SPM receiver is the simplicity which makes it an attractive technique. At the receiving end, the received data bits pass through the conventional OFDM process. Then the signal is distributed into two demodulation blocks. The first block is working as a bit detector carried by the SC power levels. This is accomplished by making the given threshold value $\mathrm{T}$ as a reference and compare it with the received power value of each SC. The threshold $\mathrm{T}$ can be defined as the power of the midpoint between high and low-level amplitudes of SCs. If the power of $\mathrm{SC}$ is higher than the threshold, it is considered as a high-power SC with naming 1 and vice versa. The second demodulator is responsible for the demodulation of conventional BPSK to the symbols [12]. 


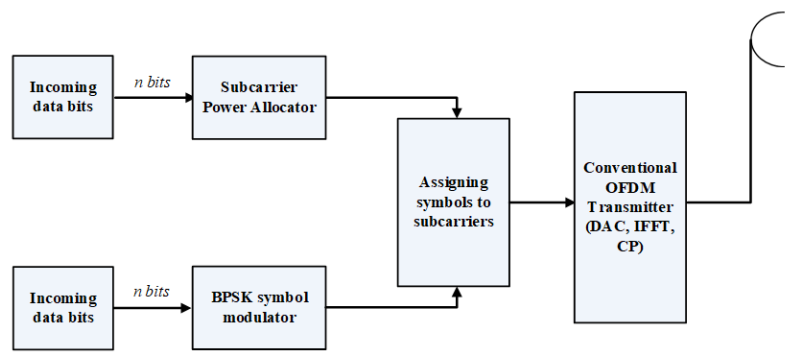

(a)

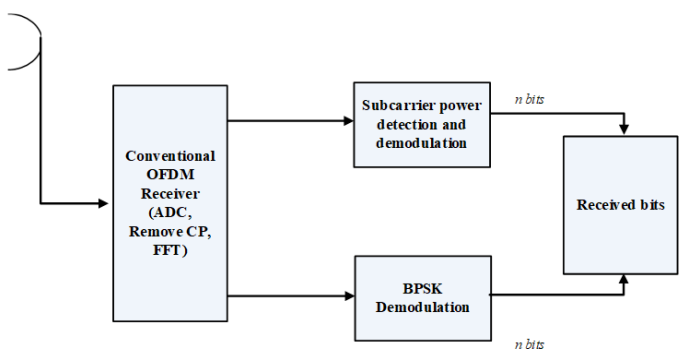

(b)

Figure 2. Block diagram of OFDM-SPM: (a) transmitter and (b) receiver

\subsection{Semiconductor optical amplifier}

Recently, optical amplifiers are playing an essential role in the telecommunication networks such as wavelength division multiplexing (WDM) systems and RoF applications. SOA has some features (e.g., low cost, small size and can be integrated with other components and modulators) that make it important in many applications [21]. Additional advantages of SOA that it has a short time response, high gain, low power, and multifunctional capabilities [22]. The below section is describing the traveling wave through SOA in VPI software [23]. Note that $L$ is the length of the device and the physical amounts are averaged over $L$ [23].

The amplifier's gain medium is depending on the carrier density $(N)$ and described by the material gain coefficient $\mathrm{g}(N)$ that is written by [24]:

$$
g(N)=\frac{d g}{d N}\left(N-N_{t r}\right)
$$

where $\frac{d g}{d N}$ represents the differential gain, which describing a slope of $g(N)$. and $N_{t r}$ is the value of carrier density at the transparency point.

The optical confinement factor $(\Gamma)$ is the key factor in SOA which determines the actual amplification of the optical waves in SOA. It can be defined as a fraction of the mode power in the active layer and by the losses of waveguide $\left(\alpha_{s}\right)$. the net gain coefficient $g_{t o t}(N)$ is given by [24]:

$$
g_{t o t}(N)=\Gamma g(N)-\alpha_{s}
$$

At each point $\mathrm{z}$ of SOA, we can calculate the total gain $G(z)$ of an optical wave as the following [23]:

$$
G(N, z)=\exp \left[g_{t o t}(N) \mathrm{z}\right]=\exp \left[\left(\Gamma g(N)-\alpha_{s}\right) \mathrm{z}\right]
$$

Assuming that the carrier density $N(z)$ is constant over the length of SOA and with using (3), the average light power $P_{a v g}$ over the SOA's length can be written as [24]:

$$
\begin{aligned}
& P_{\text {avg }}=\frac{1}{L} \int_{0}^{L} P(N, z) d z=\frac{1}{L} \int_{0}^{L} P_{\text {in }} G(N, z) d z=\frac{P_{\text {in }}}{L} \int_{0}^{L} \exp \left[g_{\text {tot }}(N) \mathrm{z}\right] d z \\
& =P_{\text {in }} \frac{\exp \left[g_{\text {tot }}(N) L\right]-1}{g_{\text {tot }}(N) L}
\end{aligned}
$$

where $P_{a v g}$ is the time function because the carrier density $N(t)$ and the input signal power are timedependent in (4). Accordingly, the dynamic equation of carrier density $N(t)$ can be expressed as [24]:

$$
\frac{d N}{d t}=\frac{I}{q V}-R(N)-\frac{\Gamma g(N) P_{a v g}(N, t) L}{V h f}
$$

where $q$ is the electron charge, $V$ is the volume which equals to $L * d * W, d$ is the thickness and $W$ is the width of the active layer and $I$ is the injection current. $h$ represents Planck's constant and $f$ is the light frequency. The recombination rate $R(N)$ consists of nonradiative transitions and spontaneous emission can be expressed with Auger recombination [25]:

$$
R(N)=A N+B N^{2}+C N^{3}
$$


where A, B, and C are constants representing different recombination processes. The (5) and (6) are solved by using the numerical method which is the fifth-order Runge-Kutta algorithm. The output optical field $E_{\text {out }}(t)$ is calculated in terms of the input field $E_{\text {in }}(t)$ and expressed as [25]:

$$
E_{\text {out }}(t)=E_{\text {in }}(t) \exp \left[\frac{(1+j \alpha) \Gamma g(N(t)) L-\alpha_{s} L}{2}\right]
$$

where $\alpha$ is the linewidth enhancement factor.

\section{SIMULATION MODEL}

Figure 3 displays the simulation model of the wavelength conversion process using SOA for OFDM-SPM modulated signal. It consists of three stages: transmitter (Tx), wavelength converter (WC), and finally the receiver $(\mathrm{Rx})$. The transmitter includes a laser diode which is considered as the main system carrier operating at wavelength $1550.116 \mathrm{~nm}(193.4 \mathrm{THz})$ and a constant laser called pump laser which is working at $1548.515 \mathrm{~nm}(193.6 \mathrm{THz})$. The electrical signal OFDM-SPM operating in E-band with carrier frequency $80 \mathrm{GHz}$ is generated to transmit a $7 \mathrm{Gbps}$ data rate. Now, laser diode signal and OFDM-SPM signal launched into Mach-Zehnder modulator (MZM) to produced one modulated signal which is called a probe signal. Then both signals; probe and pump are combined in the optical coupler (OC) with a coupling ratio (50:50) in the WC stage then injected into the SOA with an injection current (IC) of $600 \mathrm{~mA}$ in order to be transferred to the desired wavelength. Finally, the resulted optical signal will enter the receiver stage, be detected, and then de-modulated using photodetector (PD) in order to measure the performance of conversion in terms of BER, EVM, and OSNR.

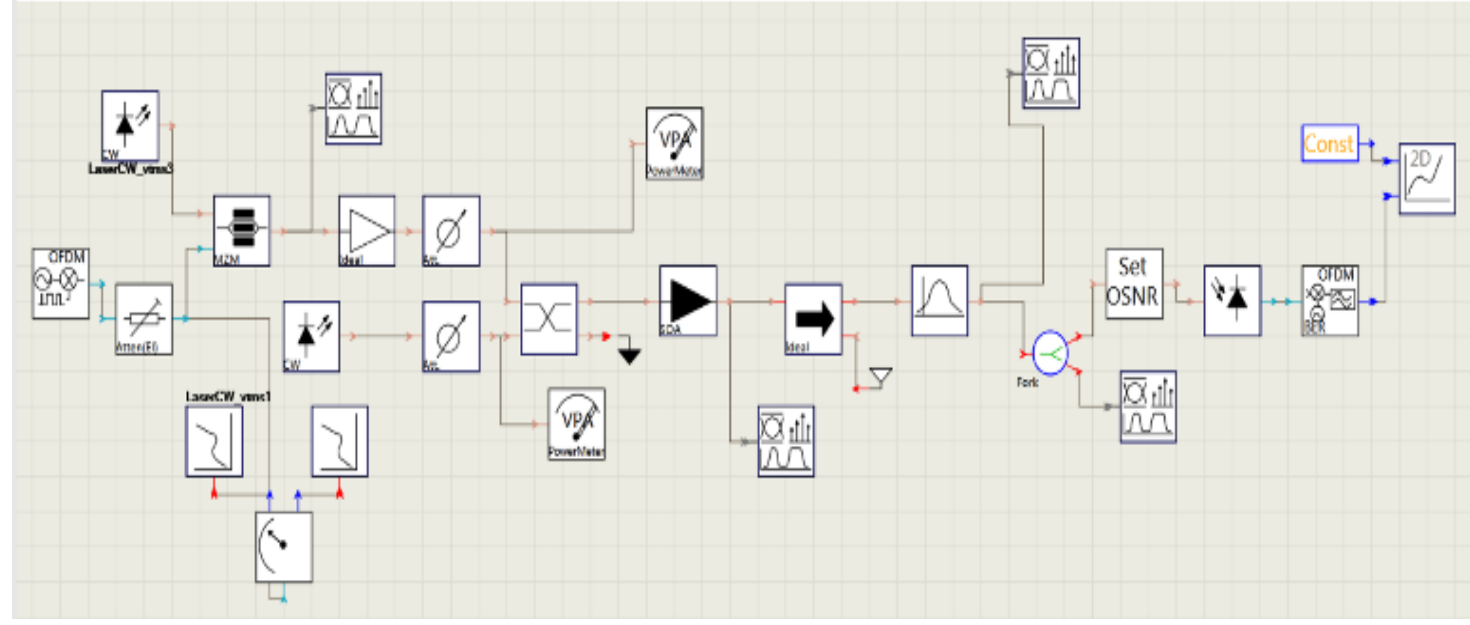

Figure 3. Simulation model for wavelength converter using SOA of OFDM-SPM signal

\section{SIMULATION RESULTS}

In this section, we will discuss the result of the proposed system. In the first step, we started the optimization of SOA's parameters to obtain the optical single-sideband (OSSB) switched signal in order to save bandwidth and reduce the chromatic dispersion (CD) when compared to double-side signal (DSB). Moreover, this optimization aims to produce a high performance of the conversion process and recommend the optimum values of system parameters.

Firstly, in order to obtain an OSSB switched signal with a high sideband suppression ratio (SSR), the injection current of SOA, power of probe, and pump signals are required to be optimized. We run the sweep option with three variables (IC, probe power, and pump power) within the allowed range then we noticed the higher SSR value to assign the optimum value of the three variables. Figure 4(a) displays how the injection current affects SSR. It can be seen that the maximum SSR achieved at 0.6 A of injection current. After that, we investigated the effects of the power of probe and pump signal on SSR, the results are shown in Figures 4(b) and 4(c) respectively. The maximum value of SSR is obtained at $9.45 \mathrm{dBm}$ of probe signal and $8.9 \mathrm{dBm}$ of pump signal.

After this optimization with the aforementioned parameters, the SOA's optical power spectrum is shown in Figure 5. As seen, the switched signal has an SSR of $22.91 \mathrm{~dB}$ which is meaning that the resulted 
switched signal is an OSSB, not a DSB. In the final step, we investigated the performance of the conversion process by using the OFDM-SPM demodulator on the receiver side. Figures 6(a) and 6(b) display the BER and EVM for the system, both figures show that the performance increased after when the value of OSNR is more than $25 \mathrm{~dB}$.

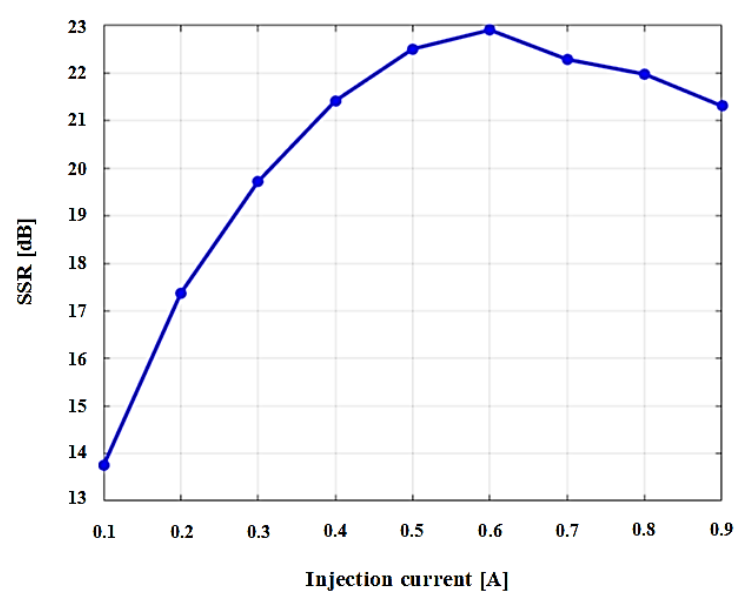

(a)

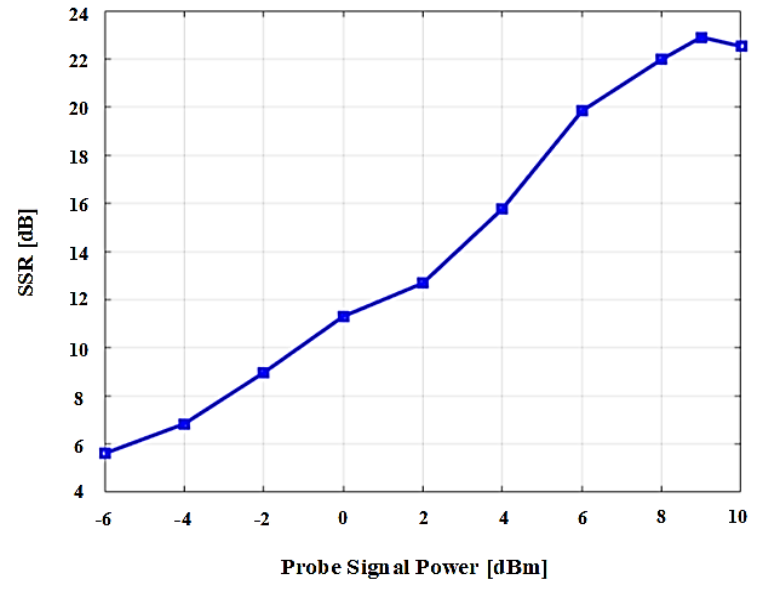

(b)

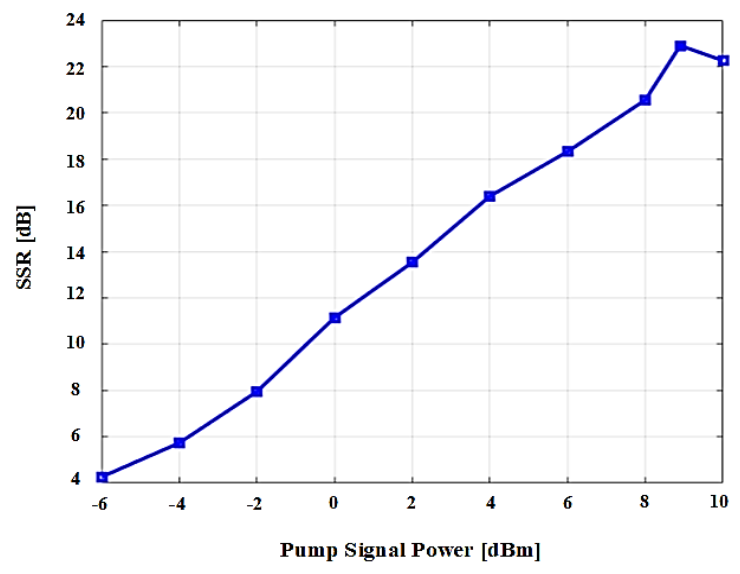

(c)

Figure 4. Effects of (a) SOA injection current, (b) probe signal power, and (c) pump signal power, on the SSR

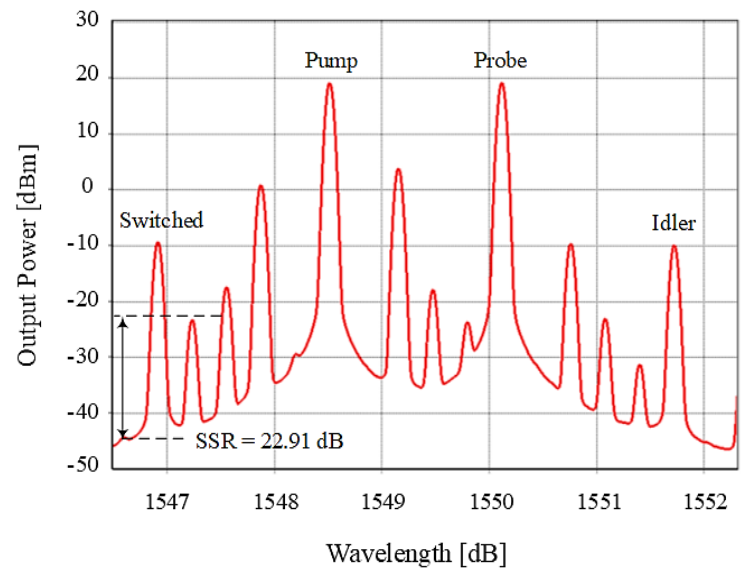

Figure 5. SOA's optical output measured spectrum for ODFM-SPM signal at $80 \mathrm{GHz}$ MMW carrier 


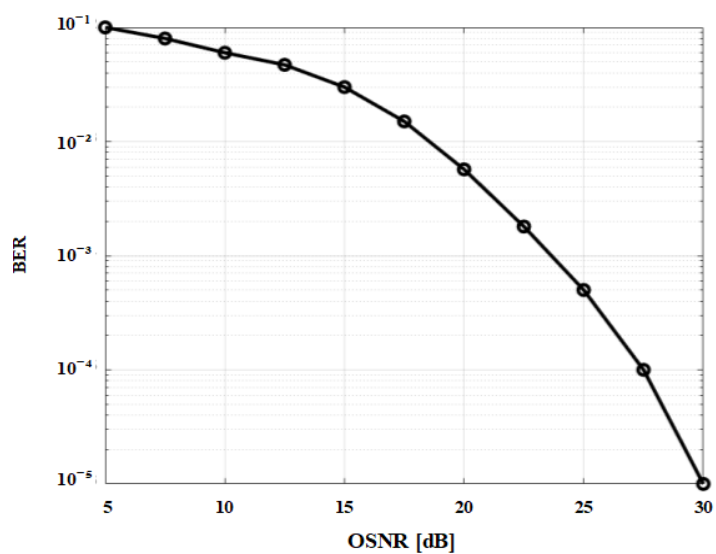

(a)

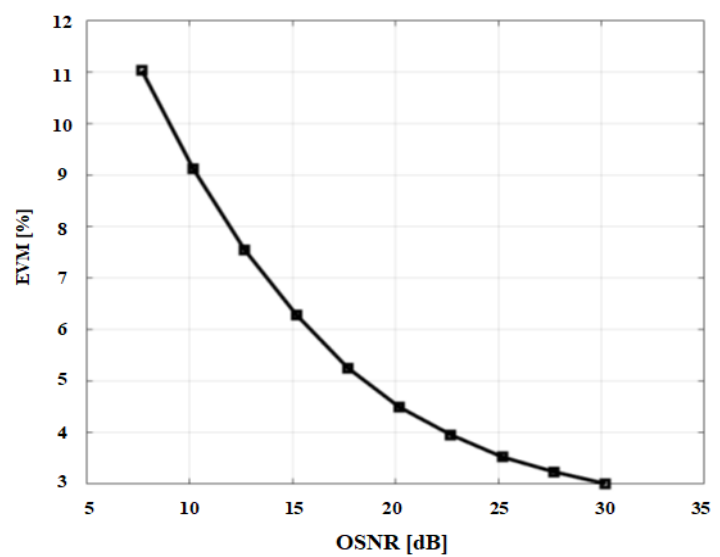

(b)

Figure 6. Switching performance in terms of (a) BER and (b) EVM

\section{CONCLUSION}

This paper demonstrated a photonic system to generate an OSSB signal with E-band carrier frequency and then convert it to the desired wavelength using SOA for OFDM-SPM modulation format. This is done using virtual photonic integrated (VPI) and MATLAB software. The fast response of the nonlinear effect of SOA (i.e., FWM and SPM) provides a fast-switching speed. A 7-Gbps OFDM-SPM signal with a carrier frequency of $80 \mathrm{GHz}$ is converted to a new wavelength and we investigated its performance by the resulted SSR which is $22.91 \mathrm{~dB}$. We optimized the main parameters affecting the SSR such as injection current of SOA, probe power, and pump power. We studied the system's performance in terms of EVM and BER. The results show that OSNR should be more than 25 to get high performance.

\section{ACKNOWLEDGEMENTS}

This work is supported by Universiti Teknologi Malaysia institutional grant 08G49 part of a collaborative project with photonic Laboratory (KACST-TIG-RFTONIC-KSU). The authors would like to thank Universiti Teknologi Malaysia and King Saud University for their cooperation.

\section{REFERENCES}

[1] A. S. Cacciapuoti, K. Sankhe, M. Caleffi, and K. R. Chowdhury, "Beyond 5G: THz-based medium access protocol for mobile heterogeneous networks," IEEE Communications Magazine, vol. 56, no. 6, pp. 110-115, Jun. 2018, doi: 10.1109/MCOM.2018.1700924.

[2] M. Shafi et al., "5G: a tutorial overview of standards, trials, challenges, deployment, and practice," IEEE Journal on Selected Areas in Communications, vol. 35, no. 6, pp. 1201-1221, Jun. 2017, doi: 10.1109/JSAC.2017.2692307.

[3] C. X. Wang, J. Bian, J. Sun, W. Zhang, and M. Zhang, "A survey of $5 \mathrm{~g}$ channel measurements and models," IEEE Communications Surveys and Tutorials, vol. 20, no. 4, pp. 3142-3168, 2018, doi: 10.1109/COMST.2018.2862141.

[4] M. Xu, J. Zhang, F. Lu, Y. Wang, D. Guidotti, and G. K. Chang, "Investigation of FBMC in mobile fronthaul networks for 5G wireless with time-frequency modulation adaptation," 2016, doi: 10.1364/ofc.2016.w3c.2.

[5] J. Zhang et al., "Full-duplex quasi-gapless carrier aggregation using FBMC in centralized radio-over-fiber heterogeneous networks," Journal of Lightwave Technology, vol. 35, no. 4, pp. 989-996, Feb. 2017, doi: 10.1109/JLT.2016.2608138.

[6] H. N. Parajuli, H. Shams, L. G. Gonzalez, E. Udvary, C. Renaud, and J. Mitchell, "Experimental demonstration of multi-Gbps multi sub-bands FBMC transmission in mm-wave radio over a fiber system," Optics Express, vol. 26, no. 6, pp. 7306-7312, Mar. 2018, doi: 10.1364/oe.26.007306.

[7] Y. Alkhlefat, A. M. Ragheb, M. A. Esmail, S. A. Alshebeili, and H. E. Seleem, "Millimeter wave switching for single carrier and aggregated filter bank multi-carrier signals in radio over fiber networks," Optical Fiber Technology, vol. 60, p. 102335, Dec. 2020, doi: 10.1016/j.yofte.2020.102335.

[8] P. Varzakas, "Optimization of an OFDM rayleigh fading system," International Journal of Communication Systems, vol. 20, no. 1, pp. 1-7, 2007, doi: 10.1002/dac.807.

[9] Z. N. Ghanim and B. M. Omran, "OFDM PAPR reduction for image transmission using improved tone reservation," International Journal of Electrical and Computer Engineering, vol. 11, no. 1, pp. 416-423, Feb. 2021, doi: 10.11591/ijece.v11i1.pp416-423.

[10] Z. M. Abid, A. A. Jafaar, and S. Q. Hadi, "PAPR reduction in OFDM system for DVB-S2," Indonesian Journal of Electrical Engineering and Computer Science, vol. 17, no. 1, pp. 317-323, Jan. 2019, doi: 10.11591/ijeecs.v17.i1.pp317-323.

[11] T. J. Rouphael, $R F$ and digital signal processing for software-defined radio. Elsevier, 2009.

[12] A. Hajar, J. M. Hamamreh, M. Abewa, and Y. Belallou, "A spectrally efficient OFDM-based modulation scheme for future wireless systems,” Apr. 2019, doi: 10.1109/EBBT.2019.8742049.

[13] F. Saadaoui, M. Fathallah, A. M. Ragheb, M. I. Memon, H. Fathallah, and S. A. Alshebeili, "Optimizing OSSB generation using semiconductor optical amplifier (SOA) for 5G millimeter wave switching," IEEE Access, vol. 5, pp. 6715-6725, 2017, doi: 10.1109/ACCESS.2017.2683064. 
[14] D. Zhu, Z. Wei, H. Wu, and S. Pan, "Photonics-based microwave switching using optical single sideband wavelength conversion in a semiconductor optical amplifier," IEEE Transactions on Microwave Theory and Techniques, vol. 65, no. 1, pp. 245-252, Jan. 2017, doi: 10.1109/TMTT.2016.2614293.

[15] G. Li, J. Li, G. Chen, and X. Huang, "SOA-based AOWC of 128QAM using gaussian pulse shaping for transmission system with 227 Gbps," Microwave and Optical Technology Letters, vol. 60, no. 9, pp. 2204-2216, Aug. 2018, doi: 10.1002/mop.31323.

[16] Y. Alkhlefat, S. M. Idrus, and F. M. Iqbal, "Numerical analysis of UFMC and FBMC in wavelength conversion for radio over fiber systems using semiconductor optical amplifier," Alexandria Engineering Journal, Nov. 2021, doi: 10.1016/j.aej.2021.10.059.

[17] K. Digambar and R. K. Jeyachitra, “An efficient photonic-based millimeter wavelength switching techniques towards 5G," Mar. 2018, doi: 10.1109/WiSPNET.2018.8538742.

[18] S. H. Lee and J. I. Song, "An XPolM-based all-optical SSB frequency up-conversion technique in an SOA," IEEE Photonics Technology Letters, vol. 29, no. 7, pp. 627-630, Apr. 2017, doi: 10.1109/LPT.2017.2676122.

[19] Y. A. Alkhlefat, A. Ragheb, M. A. Esmail, and S. Alshebeili, "Millimeter wave switching in radio over fiber networks using semiconductor optical amplifier (SOA)," Apr. 2018, doi: 10.1109/NCG.2018.8593081.

[20] A. M. Jaradat, J. M. Hamamreh, and H. Arslan, "Modulation options for OFDM-based waveforms: classification, comparison, and future directions," IEEE Access, vol. 7, pp. 17263-17278, 2019, doi: 10.1109/ACCESS.2019.2895958.

[21] D. Huang, "Semiconductor optical amplifiers and related applications," in Applications of Photonic Technology 5, Feb. 2003, vol. 4833, doi: 10.1117/12.478235.

[22] A. Bogoni, L. Potì, C. Porzi, M. Scaffardi, P. Ghelfi, and F. Ponzini, "Modeling and measurement of noisy SOA dynamics for ultrafast applications," IEEE Journal on Selected Topics in Quantum Electronics, vol. 10, no. 1, pp. 197-205, Jan. 2004, doi: 10.1109/JSTQE.2003.822919.

[23] S. Shimada and H. Ishio, Optical Amplifiers and Their Applications. Chichester: 1st Ed., John Wiley, 1994.

[24] M. J. Adams, H. J. Westlake, M. J. O'mahony, and I. D. Henning, “A comparison of active and passive optical bistability, in semiconductors," IEEE Journal of Quantum Electronics, vol. 21, no. 9, pp. 1498-1504, Sep. 1985, doi: 10.1109/JQE.1985.1072818.

[25] Govind Agrawal, Long-Wavelength Semiconductor Lasers, 1st ed. Springer Netherlands, 1986.

\section{BIOGRAPHIES OF AUTHORS}
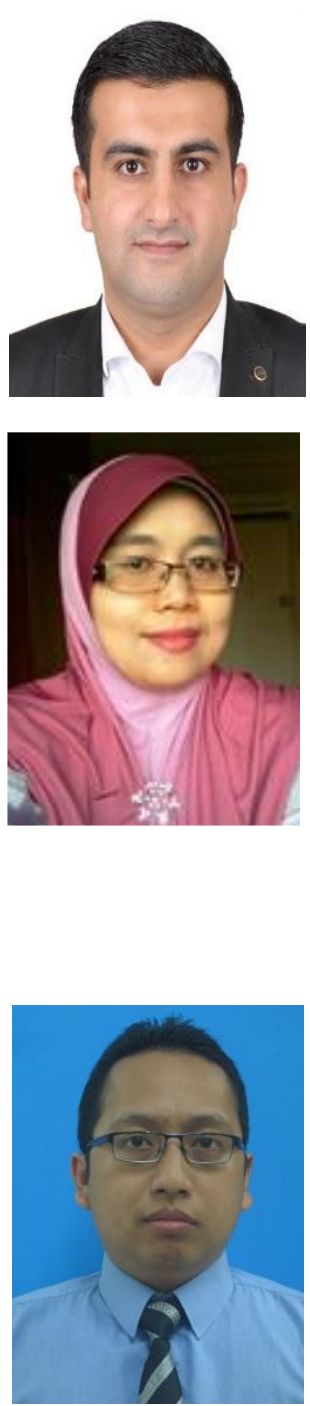

Yazan Alkhlefat (D) SC P Sas born in Jordan on March 30, 1988. He received his B.S. degree from AL Yarmouk University, Irbid, Jordan, in 2011, the master's degree from King Saud University, Riyadh, Saudi Arabia, in 2019, both in electrical engineering. He is currently a Ph.D. student at Universiti Teknologi Malaysia (UTM) in electrical engineering as well. He was working as a NOC Engineer at STC Operator, Saudi Arabia, from 2011 to 2012. He was working as a Microwave Transmission design and optimization Engineer at HUAWEI, for 4G projects at STC Operator, Saudi Arabia, From 2013-2018. Currently, he is working as a Senior Microwave Transmission design and optimization Engineer at NOKIA, Saudi Arabia. He is interested in research areas such as photonic systems, optical fiber communications, 5G modulation formats, $\mathrm{THz}$ communications, and radio over fiber (RoF). He can be contacted at email: amyazan1988@graduate.utm.my.

Sevia Mahdaliza Idrus (D) SC P is the Deputy Dean (Development and Alumni), Faculty of Engineering, UTM. She received her Bachelor in Electrical Engineering in 1998 and Master in Engineering Management in 1999, both from UTM. She obtained her Ph.D. in 2004 from the University of Warwick, United Kingdom in optical communication engineering. She has served UTM since 1998 as an academic and administrative staff. Her main research interests are optical communication system and network, optoelectronic design, and engineering management. Her research output has been translated into a number of publications $(\mathrm{H}-$ indexed-15) and IPR including over 200 refereed research papers, 10 patents granted, 30 patent filings and holds 36 UTM copyrights. To date, she has secured and been involved in 38 research and consultation projects with a total value of USD22M. She is actively involved in a number industrial and international research collaboration projects, delivered keynote and invited speeches to many international conferences and seminars. She is Senior Member of IEEE and member of Editorial Board of few refereed international journals. She has been appointed as Guest Professor at Osaka Prefecture University and Tokai University, Japan in 2011 and 2014, respectively. He can be contacted at email: sevia@utm.my.

Farabi M. Iqbal (iD) $\mathrm{SC}$ P is a senior lecturer in the school of electrical engineering at Universiti Teknologi Malaysia, Malaysia where he has been a faculty member since 2009 . He holds a M.Eng. from Universiti Teknologi Malaysia, and a Ph.D. from Delft University of Technology, The Netherlands. His main research interests revolve around network routing, resiliency, and optimization. He can be contacted at email: alfarabi@utm.my. 